\title{
Traduire
}

Revue française de la traduction

234 | 2016

La traduction, un sport de haut niveau

\section{Les anglicismes dans les sports de glisse}

\section{Elisabeth Monrozier}

\section{(2) OpenEdition}

\section{Journals}

Édition électronique

URL : http://journals.openedition.org/traduire/808

DOI : 10.4000/traduire.808

ISSN : 2272-9992

\section{Éditeur}

Société française des traducteurs

Édition imprimée

Date de publication : 15 juin 2016

Pagination : 69-74

ISSN : 0395-773X

\section{Référence électronique}

Elisabeth Monrozier, "Les anglicismes dans les sports de glisse », Traduire [En ligne], 234 | 2016, mis en ligne le 15 juin 2018, consulté le 05 mai 2019. URL : http://journals.openedition.org/traduire/808 ; DOI : 10.4000/traduire.808 


\title{
Les anglicismes dans les sports de glisse
}

\author{
Elisabeth Monrozier
}

Comme toute spécialisation, la traduction sportive, que nous étudierons ici de l'anglais vers le français, pose au traducteur un certain nombre de questions et de défis, qu'il devra tenter de résoudre en s'appuyant tant sur sa maîtrise linguistique que sur sa connaissance du milieu. Dans le domaine des sports de glisse, la prolifération des anglicismes en français représente un problème particulièrement épineux, alors que le traducteur pointilleux cherche généralement à les éviter à tout prix et ne peut se résoudre à les intégrer dans sa traduction sans un sentiment d'échec, voire de trahison. Entre la tentation de traduire tous les termes en " bon français " et celle de multiplier les anglicismes, il doit faire preuve de discernement et d'équilibre pour que le texte cible réponde aux impératifs d'intelligibilité et de naturel.

\section{Inévitables anglicismes}

Nous traiterons dans cet article de trois grandes familles de sports de glisse, correspondant à autant d'environnements de pratique : glisse urbaine (skate, roller), nautique (surf, windsurf) ou sur neige (ski, snowboard). Leur attirance pour les anglicismes découle à la fois de l'origine de ces sports et de leur philosophie. Certes, le surf est né il y a plusieurs siècles à Hawaï, et si l'on remonte ne serait-ce qu'une vingtaine d'années en arrière, le langage du ski semble dénué d'emprunts à l'anglais, puisque cette discipline est arrivée en France au début du xxe siècle en provenance directe de Scandinavie. C'est pourtant bel et bien aux États-Unis que l'on doit la diffusion du surf, puis l'invention progressive des "nouvelles glisses ", essentiellement dans les années quatre-vingt et quatre-vingt-dix. Le renouveau du ski est également venu d'outre-Atlantique, avec un accent mis non plus sur la vitesse, mais sur les figures acrobatiques (freestyle) et le ski hors-piste (freeride). Face à cette abondante terminologie anglaise, il aurait été possible de proposer ou imposer d'en haut des termes français, entre autres par l'intermédiaire d'une fédération sportive nationale ; mais ces sports ont comme point commun une philosophie de liberté et d'indépendance qui leur fait éviter toute forme de 
structure et d'organisation. Dès lors, les recommandations terminologiques n'ont aucune prise sur cet univers. Autre aspect fondamental de l'état d'esprit propre à ces sports : la décontraction, qui semble devoir s'exprimer prioritairement en anglais. Comment avoir l'air décontracté pardon, cool - sans un usage intensif de termes anglais ? Question de point de vue, bien sûr ! Ajoutons à cela l'esprit de communauté de la glisse, porteuse d'une identité forte. Celle-ci s'exprime dans un certain nombre d'habitudes partagées : l'emploi d'un langage spécifique, opaque au monde extérieur, en fait partie, au même titre que la tenue vestimentaire ou la musique. En utilisant des anglicismes bien choisis, le traducteur favorise la reconnaissance de l'auteur comme membre de cette communauté et, par conséquent, garantit une meilleure réception du message par le lecteur. Sélectionner systématiquement des termes français recommandés par exemple par la Délégation générale à la langue française et aux langues de France (DGLFLF) ferait passer le locuteur pour un étranger à ce groupe, éloigné des idéaux de la glisse et, forcément, ringard et obtus.

Ces nombreux anglicismes ne sont pourtant pas sans poser problème. Avant même le désir de protection et d'enrichissement de la langue française, souvent propre au traducteur, il faut viser en priorité la clarté de la langue pour le lecteur, sans quoi la traduction n'atteint pas son objectif de communication. Le risque de confusion n'est pas négligeable : le francophone ne maîtrisant pas l'anglais et sa syntaxe aura vite fait de mélanger les shortboards, planches de surf courtes, avec des boardshorts, maillots de bain conçus pour la pratique du surf. Dans d'autres cas, la rencontre avec un anglicisme suscite l'incompréhension la plus complète, alors qu'un terme français préexistant ou créé pour répondre à ce besoin serait parfaitement clair. Le terme " marche arrière " est par exemple plus explicite que fakie, qui fait pourtant autorité dans l'usage.

On distingue maintenant l'étroite ligne de crête sur laquelle le traducteur devra évoluer, entre impératif de compréhension et usage en vigueur chez le public destinataire du message.

Un autre paramètre vient parfois orienter ou, dans le pire des cas, entraver les décisions terminologiques du traducteur : les consignes du client. Celui-ci peut fournir un glossaire complet ou quelques termes clés pour lesquels l'usage varie. C'est le plus souvent un gain de temps pour le traducteur, mais des termes mal choisis ou erronés le conduiront à discuter parfois longuement avec le commanditaire.

\section{Les différentes options}

À partir de ce constat, le traducteur se trouve face à trois possibilités. La première correspond à la situation idéale, où le terme a un équivalent français en usage. Le traducteur peut l'adopter sans hésitation et n'a pas à redouter les problèmes de compréhension. 
Le deuxième cas est celui de la cohabitation d'un anglicisme et d'un terme français pour traduire le même terme source dans le même domaine. Pour favoriser la compréhension du texte, il est recommandé de sélectionner le terme français. On trouve dans cette catégorie la figure de skate portant le nom de 50-50, qui se lit indifféremment fifty-fifty ou cinquante-cinquante. De même, les skis de freestyle présentant une spatule, c'est-à-dire une extrémité relevée, à l'avant et à l'arrière, sont qualifiés aussi bien de twin-tip que de bispatulés. On rencontre encore cette coexistence de deux termes synonymes dans la désignation des skis avec rocker ou cambre inversé, ou dans le couple combinaison/wetsuit.

Dans un troisième type de situation, aucun terme français n'est attesté, c'est l'anglicisme qui prévaut. Le terme anglais est alors repris à l'identique dans la traduction. Si cet anglicisme est courant, suffisamment transparent, ou si le public visé est très averti, le traducteur peut se passer d'explication. En revanche, en cas de doute sur la facilité de compréhension du terme, il est judicieux de donner des éclaircissements - traduction ou paraphrase - entre parenthèses ou en bas de page à la première occurrence.

\section{Polymorphisme des anglicismes}

Le traducteur doit être vigilant lors du choix du terme, car les pièges sont nombreux. En effet, ce n'est pas parce que le terme anglais de départ semble utilisé tel quel en français qu'il peut l'être dans toutes les circonstances. Selon le contexte, un même terme devra être traduit ou restera en anglais. Voyons quels facteurs peuvent être déterminants dans ce choix.

Les variantes géographiques du français donnent toujours lieu à des divergences dans l'utilisation des anglicismes. Si tout le monde semble vouloir y échapper, nul n'y parvient vraiment et il serait audacieux de pointer telle ou telle variante comme moins perméable aux emprunts étrangers. II faudra donc vérifier au cas par cas quel est l'usage répandu dans la région du monde visée. On observe par exemple que les Canadiens francophones ont largement adopté le terme de planche à neige, même s'il ne fait pas l'unanimité, alors qu'il est inexistant en France où on lui préfère le terme de snowboard (ou snow), malgré les recommandations de la DGLFLF. En revanche, un autre moyen de locomotion sur neige obéit au mécanisme inverse : la motoneige des Français est nommée "skidoo " au Canada.

Une autre dimension propice au passage d'une catégorie à l'autre est la dimension temporelle. L'évolution est souvent la même : un sport relativement ancien, désigné sous une appellation française, revient sur le devant de la scène, rafraîchi par un nouveau nom... en anglais. C'est ainsi que le patin à roulettes est devenu " roller " après avoir aligné ses quatre roues, et que la planche à voile se transforme progressivement en " windsurf ". On remarque par ailleurs que cette évolution terminologique peut conduire à remplacer un anglicisme par un autre : le terme "surf des neiges ", par exemple, a disparu au profit de "snowboard". 
Intéressons-nous maintenant à l'importance de la discipline dont il question (s'il est permis de parler de "disciplines " pour des activités synonymes de liberté et de recherche de sensations). Les sports de glisse étant une grande famille, ils ont déjà en commun une partie de leur vocabulaire et un certain nombre d'anglicismes se retrouvent dans plusieurs d'entre eux, avec un sens et un emploi très proches. Les termes spot, trip, ride, shape et beaucoup d'autres sont familiers dans tout l'univers des sports de glisse.

D'autres termes, en revanche, ne se transposent pas sur le même mode d'un sport à l'autre, alors qu'ils désignent des réalités semblables. Prenons par exemple la figure consistant à sauter en réalisant un tour complet sur soi-même. Réalisée avec un skate, elle s'appelle un three-sixty, mais si le sportif est chaussé de skis, c'est un trois-six. Dans le même ordre d'idée, les skateurs utilisent pour s'élancer et réaliser leurs figures acrobatiques des rampes, structures artificielles ayant la forme d'un demi-cylindre, quand les skieurs et snowboardeurs se servent d'un half-pipe, installation similaire, mais réalisée en neige. On observe aussi que plusieurs pratiques nécessitent de relier la planche à la chaussure ou à la cheville de l'utilisateur, pour éviter de perdre le matériel ou de le transformer en projectile incontrôlable en cas de chute. Bien que le concept soit le même, l'usage varie selon le sport : les surfeurs appellent ce dispositif un leash, mais les amateurs de ski de randonnée l'appellent soit "lanière ", soit... leash.

Ces différences de traitement entre les sports se retrouvent jusque dans les branches d'un même sport, ce qui risque d'ajouter à la confusion du traducteur. Ainsi, il semblerait normal que la structure qui permet à un skieur de sauter porte toujours le même nom, mais dans la réalité il faut distinguer le tremplin, conçu pour le saut à ski, du kicker ou big air, destiné aux figures acrobatiques du ski freestyle.

\section{Une grammaire particulière}

Attention, le genre grammatical peut être différent de celui attendu, c'est-à-dire du genre du mot français qui aurait pu le traduire. Ainsi l'anglais team, normalement traduit par équipe, féminin, devrait devenir " la " team. On trouve pourtant plus souvent le masculin, notamment pour désigner un " team de pro riders " : il ne s'agit pas de compétiteurs réunis pour représenter leur ville ou leur pays, mais d'ambassadeurs d'une marque, sportifs talentueux participant aussi à la conception du matériel. À noter cependant que l'usage hésite sur le genre du mot "team ". Si l'on revient au terme leash, on constate qu'il est toujours masculin en français alors qu'il ne remplace que des mots féminins : attache, lanière, sangle... La prudence est de mise encore une fois lors du recours à la forme abrégée d'un terme, car le genre peut changer en cours de route : ainsi le snowboard, indubitablement masculin, devient-il une board.

Remarquons aussi que l'anglicisme en usage n'est pas toujours pur, il peut être mâtiné de français d'une façon ou d'une autre, ce qui complique son emploi. Ainsi, tous les verbes 
directement issus de l'anglais doivent se conjuguer et sont donc affublés de terminaisons françaises : je switche, tu grabais, il ridera... Certains anglicismes ont donné naissance à des dérivés après adjonction d'un suffixe, par exemple " glasseur " et " glassage ". On trouve enfin des néologismes combinant un anglicisme et un terme français, comme avec le terme de "freerando ", désignant le ski de randonnée axé prioritairement sur la recherche de belles descentes.

Cet aperçu forcément limité du paysage terminologique des sports de glisse met en lumière un principe incontournable : il n'existe pas de règle absolue en la matière. Pour choisir et employer correctement l'anglicisme qui facilitera la réception du message au lieu de la gêner, le meilleur glossaire ne remplacera pas les recherches minutieuses. Le traducteur devra se documenter sur le domaine concerné et examiner chaque terme pressenti, en veillant à consulter des ressources récentes et, de préférence, rédigées par des pratiquants du sport traité. Les blogs, forums et réseaux sociaux sont donc souvent les sources les plus fiables et peuvent être corroborés auprès des habitués des skateparks ou des pistes de ski. Autant de bonnes habitudes qui peuvent s'appliquer à la traduction dans d'autres secteurs.

elisabeth.monrozier@yahoo.fr

Traductrice libérale de l'anglais et du russe vers le français depuis 2010 après deux ans de salariat, Elisabeth Monrozier se spécialise dans le sport et l'éducation. Elle est titulaire du Master de traduction éditoriale, économique et technique de l'ESIT (2007), où elle a commencé à se spécialiser en rédigeant un mémoire sur la terminologie du matériel de ski alpin. www.grandslamtranslation.com 


\section{CHACUN SON TOUR...}
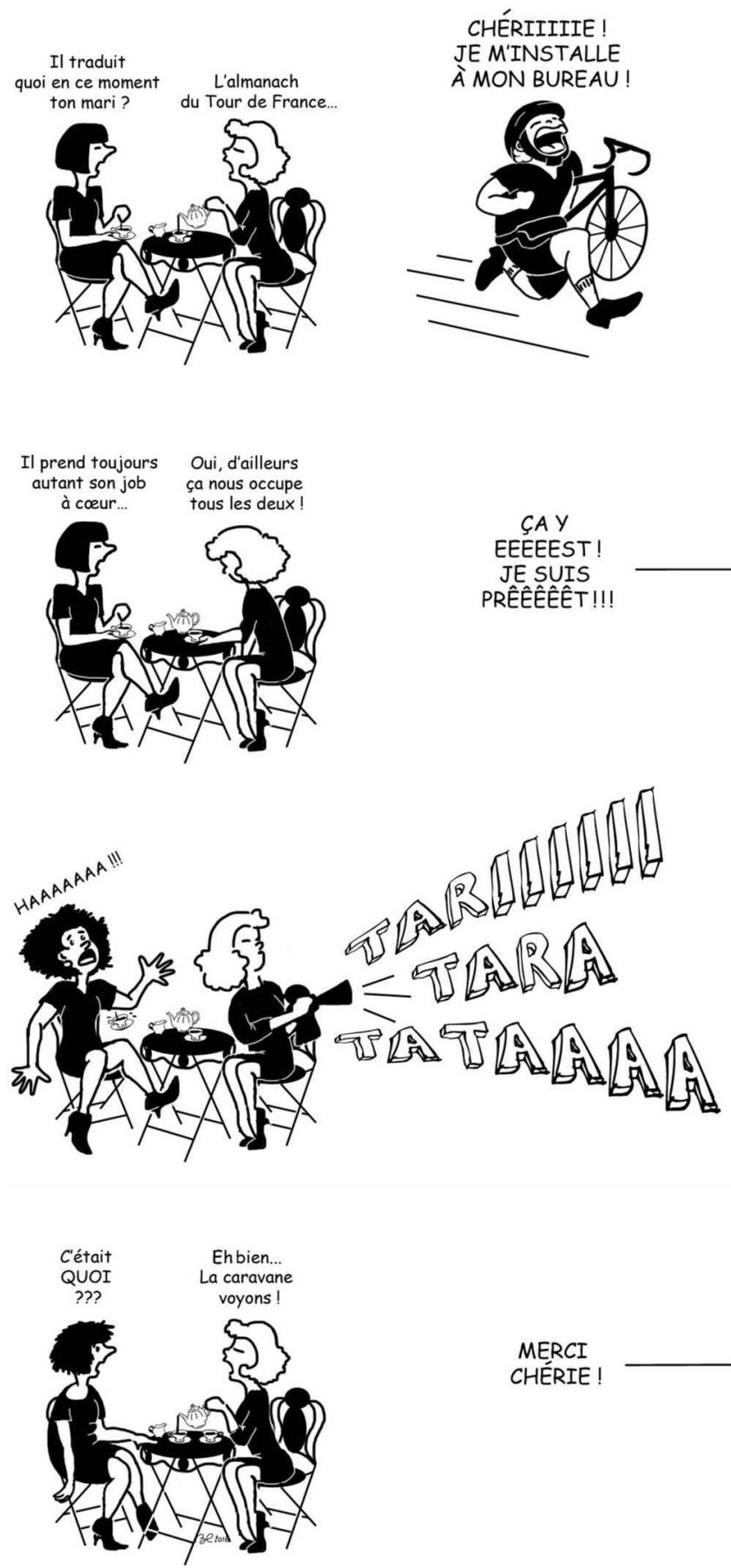